\title{
Sigles et abréviations
}

AEA : The Albert Einstein Archives at the The Hebrew University of Jerusalem.

AEG : Allgemeine Elektricitäts-Gesellschaft.

AISC : Association internationale des sociétés chimiques.

CA : Commission administrative (de l'IIPS).

CPAE : The Collected Papers of Albert Einstein (Einstein Papers project, Caltech, volumes publiés par Princeton University Press).

CSI : Comité scientifique international (de l'IIPS).

ESPCI : École supérieure de physique et de chimie industrielles de la Ville de Paris.

ETH (Zurich) : Eidgenössische Technische Hochschule (Zurich).

FIS : Fonds des Instituts Solvay.

IICS : Institut international de chimie Solvay.

IIPCS : Instituts internationaux de physique et de chimie fondés par Ernest Solvay.

IIPS : Institut international de physique Solvay.

IUPAC : International Union of Pure and Applied Chemistry.

IUPAP : International Union of Pure and Applied Physics.

KNAW : Koninklijke Nederlandse Akademie van Wetenschappen.

KWG : Kaiser-Wilhelm-Gesellschaft zur Förderung der Wissenschaften.

NHA : Noord-Hollands Archief, Haarlem.

PSL : Paris Sciences et Lettres. Pôle de recherche et d'enseignement supérieur.

PTR : Physikalisch-Technische-Reichsanstalt.

RMB : Rijksmuseum Boerhaave à Leiden.

S.a.b.ULB : Service des archives et des bibliothèques de l'Université libre de Bruxelles.

SCB : Société chimique de Belgique.

Solvay I : Premier Conseil de physique Solvay (1911).

Solvay II : Deuxième Conseil de physique Solvay (1913).

Solvay III : Troisième Conseil de physique Solvay (1921).

Solvay IV : Quatrième Conseil de physique Solvay (1924).

Solvay V : Cinquième Conseil de physique Solvay (1927).

Solvay VI : Sixième Conseil de physique Solvay (1930).

ULB : Université libre de Bruxelles. 
